\title{
MÉTODO SHORTCUT PARA OBTENÇÃO DE MODELOS DINÂMICOS SIMPLIFICADOS PARA COLUNAS DE DESTILAÇÃO
}

\author{
B. E. BUENO ${ }^{1}$, J. O. TRIERWEILER ${ }^{2}$ e P. R. B. FERNANDES ${ }^{3}$
}

1,2,3 Universidade Federal do Rio Grande do Sul, Departamento de Engenharia Química E-mails para contato: brunoettori@gmail.com ${ }^{1}$, jorge@enq.ufrgs.br ${ }^{2}$, pedro@enq.ufrgs.br ${ }^{3}$

RESUMO - Um método para obter modelos dinâmicos reduzidos para colunas de destilação, de diferentes graus de pureza para misturas binárias aproximadamente ideais, é proposto. Expressões analíticas aproximadas, para as constantes de tempo de cada função de transferência FT, foram desenvolvidas com base no Método das Potências com deflação em conjunto com estimativas de bandas limites para autovalores. A ordem de cada FT, determinada com base na determinação dos modos dominantes, e as expressões analíticas para seus zeros foram desenvolvidas com base no método de redução de Litz. Com esta estrutura, obtêm-se modelos dinâmicos shortcut a partir de parâmetros de operação da coluna.

\section{INTRODUÇÃO}

A destilação é o mais importante processo físico de separação na indústria química e petroquímica, devido à sua tecnologia bem estabelecida e sua relativa simplicidade. Apesar do compromisso inerente entre a eficiência de separação, para um número determinado de estágios da coluna, e o consumo energético do processo, essa operação unitária é a mais econômica e eficiente para a obtenção de produtos com determinada pureza, em escala industrial.

Algumas características de modelos de processos de destilação, em seu formato rigoroso, dificultam a sua simulação dinâmica. A não linearidade característica do perfil de composição destes sistemas, que decorre do equacionamento do equilíbrio líquido-vapor em cada estágio, é associada com as taxas de variação das equações diferenciais do modelo com respeito aos estados ao longo dos perfis destes estados. O acoplamento entre os estados correspondentes às composições molares, devido à interação entre um determinado estágio e os adjacentes, implica efeitos de reciclo (feedback) no sistema que deslocam os polos deste. Conforme a pureza da coluna aumenta, o polo dominante é direcionado para valores menos elevados em módulo, correspondentes a maiores constantes de tempo. Este efeito é identificado mediante as respostas dinâmicas mais lentas de colunas de elevada pureza em comparação com as de operações em baixa pureza. Com isto, para o modelo linearizado, a razão entre os valores absolutos do maior autovalor e do menor autovalor aumenta, podendo reduzir o desempenho de métodos numéricos sensíveis à rigidez do sistema. 
As características citadas limitam a utilização de simulações dinâmicas rigorosas em tempo real. Adicionalmente, para determinadas aplicações é suficiente um modelo simplificado que capture as características dinâmicas essenciais do sistema em amplas faixas operacionais. Tais aplicações contemplam principalmente controle por feedback, controle preditivo linear e não linear e o desenvolvimento de ferramentas para treinamento de operadores.

A motivação do desenvolvimento de modelos simplificados para colunas de destilação provém da observação de que a resposta dinâmica das composições molares é similar à característica de um modelo de baixa ordem, independentemente da quantidade de estágios do equipamento. $\mathrm{O}$ trabalho de Levy et. al (1969) verificou a dinâmica dominante das composições molares frente aos aspectos hidráulicos e térmicos. Como a formulação de modelos dinâmicos simplificados para destilação tem sido um importante campo de pesquisa, foram desenvolvidos tanto modelos lineares, como os formulados por Skogestad e Morari (1987), como não lineares, como o de Bernallou (1986).

O presente trabalho propõe um método de geração de modelos dinâmicos analíticos simplificados para colunas de destilação binárias de comportamento relativamente próximo ao ideal. O método é concebido para que os modelos possam ser gerados a partir de simulações estacionárias, como funções de variáveis relevantes para sua caracterização e atualizados a partir de dados do processo. Para amplas faixas de pontos de operação, os modelos devem capturar o comportamento dinâmico essencial do respectivo sistema.

\section{SISTEMA ESTUDADO E MODELOS REPRESENTATIVOS}

São abordadas colunas de destilação de alta, média e baixa pureza, considerando-se um número fixo de pratos, para distintos cenários de operação. Assume-se que as colunas são destinadas a separar misturas binárias com comportamento relativamente próximo ao ideal, alimentadas como líquido saturado.

\subsection{Hipóteses Simplificativas e Modelo Não Linear}

Consideram-se as seguintes hipóteses simplificativas: holdup da fase vapor desprezível frente ao da fase líquida; holdup $M$ constante da fase líquida (subíndices $C$ e $R$ para condensador e refervedor, respectivamente); vazões molares constantes em cada seção da coluna ( $V$ e $L$, onde os subíndices $R$ e $E$ correspondem às seções de retificação e esgotamento, respectivamente); volatilidade relativa $\alpha$ constante ao longo da coluna. Destas, decorre o modelo não linear:

$$
\begin{array}{ll}
M_{C} \frac{d x_{i, 1}}{d t}=V_{R} y_{i, 2}-L_{R} x_{i, 1}-D y_{i, 1} & j=1 \\
M \frac{d x_{i, j}}{d t}=V_{R} y_{i, j+1}+L_{R} x_{i, j-1}-V_{R} y_{i, j}-L_{R} x_{i, j} & 1<j<f-1 \\
M \frac{d x_{i, j}}{d t}=V_{E} y_{i, j+1}+L_{R} x_{i, j-1}-V_{R} y_{i, j}-L_{R} x_{i, j}+(1-q) F y_{i, F} & j=f-1
\end{array}
$$




$$
\begin{array}{ll}
M \frac{d x_{i, j}}{d t}=V_{E} y_{i, j+1}+L_{R} x_{i, j-1}-V_{E} y_{i, j}-L_{E} x_{i, j}+q F x_{i, F} & j=f \\
M \frac{d x_{i, j}}{d t}=V_{E} y_{i, j+1}+L_{S} x_{i, j-1}-V_{E} y_{i, j}-L_{E} x_{i, j} & f<j<n \\
M_{R} \frac{d x_{i, n}}{d t}=L_{E} x_{i, n-1}-V_{E} y_{i, n}-B x_{i, n} & j=n \\
D=V_{R}-L_{R} ; B=L_{E}-V_{E} & \\
V_{R}=V+(1-q) F ; V_{E}=V & \\
L_{R}=L ; L_{E}=L+q F & \\
y_{i, j}=\frac{\alpha x_{i, j}}{1+(\alpha-1) x_{i, j}} &
\end{array}
$$

\subsection{Modelo Linear}

Linearizando-se o modelo não linear em torno de um ponto de operação estacionário simbolizado por $s s$, obtém-se a matriz Jacobiana em relação aos estados $A_{C}$. Os elementos de $B_{C}$, a matriz Jacobiana de entradas como variáveis manipuladas $(M V=L$ ou $M V=V)$, e os de $A_{C}$, são definidos por:

$$
\left.a_{j, k} \equiv \frac{\partial\left(\frac{d x_{i, j}}{d t}\right)}{\partial x_{i, k}}\right|_{s s} ;\left.b_{j, M V} \equiv \frac{\partial\left(\frac{d x_{i, j}}{d t}\right)}{\partial M V}\right|_{s S}
$$

As variáveis $\operatorname{In} D_{1_{i, j}}, \operatorname{In} U_{1_{i, j}}$ e $O u t_{1, j}$, correspondem aos elementos de $A_{C R}$ :

$$
A_{C R}=I n D_{1}+\operatorname{InU}_{1}+O u t_{1}=\frac{M}{D} A_{C}
$$

As razões $R_{L D}$ e $R_{D F}$ são importantes para a análise dinâmica das colunas:

$$
\begin{aligned}
& R_{L D}=\frac{L_{R}}{D} ; \quad R_{D F}=\frac{D}{F} \\
& \operatorname{InD}_{1_{1,1}}=-\frac{M}{M_{C}}\left(R_{L D}+1\right)\left(\frac{\left.\alpha x_{i, 2}\right|_{S S}}{1+\left.(\alpha-1) x_{i, 2}\right|_{S S}}\right) \quad ; O u t_{1,1}=\frac{M}{M_{C}}\left(R_{L D}+\frac{\left.\alpha x_{i, 1}\right|_{S S}}{1+\left.(\alpha-1) x_{i, 1}\right|_{S S}}\right) \\
& \operatorname{InU}_{1_{n, n}}=-\frac{M}{M_{R}}\left(R_{L D}+\frac{q}{R_{D F}}\right)\left(\frac{\left.\alpha x_{i, 2}\right|_{s s}}{1+\left.(\alpha-1) x_{i, 2}\right|_{s s}}\right) ; O u t_{1 n, n}=\frac{M}{M_{R}}\left(\frac{1}{R_{D F}}-1+\left(R_{L D}+1\right) \frac{\left.\alpha x_{i, n}\right|_{s s}}{1+\left.(\alpha-1) x_{i, n}\right|_{s s}}\right) \\
& \text { Out }_{1 j, j}=R_{L D}+\left(R_{L D}+1\right)\left(\frac{\left.\alpha x_{i, j}\right|_{s s}}{1+\left.(\alpha-1) x_{i, j}\right|_{s S}}\right) \quad 1<j \leq f-1
\end{aligned}
$$




$$
\begin{array}{ll}
\text { Out }_{1_{j, j}}=\left(R_{L D}+1-\frac{1-q}{R_{D F}}\right)\left(\frac{\left.\alpha x_{i, j}\right|_{s s}}{1+\left.(\alpha-1) x_{i, j}\right|_{S S}}\right)+R_{L D}+\frac{q}{R_{D F}} & f \leq j<n \\
\operatorname{InD}_{1_{j, j+1}}=-\left(R_{L D}+1\right)\left(\frac{\left.\alpha x_{i, j}\right|_{S S}}{1+\left.(\alpha-1) x_{i, j}\right|_{S S}}\right) & 1<j<f-1 \\
\operatorname{InD}_{1_{j, j+1}}=-\left(R_{L D}+1-\frac{1-q}{R_{D F}}\right)\left(\frac{\left.\alpha x_{i, j}\right|_{S S}}{1+\left.(\alpha-1) x_{i, j}\right|_{S S}}\right) & f-1 \leq j<n \\
\operatorname{In} U_{1_{i, i+1}}=-R_{L D}, 1<i \leq f ; \quad \operatorname{In} U_{1_{i, i+1}}=-R_{L D}-\frac{q}{R_{D F}}, & f<i<n
\end{array}
$$

Para $A_{C}$, onde $x_{j}$ corresponde à composição de saída da respectiva FT e $x_{k}$ à entrada, e para $B_{C}$ :

$$
G_{x_{j}, x_{k}}(\mathrm{~s})=\frac{a_{j, k}}{s-a_{j, j}} ; \quad G_{x_{j}, M V}(\mathrm{~s})=\frac{b_{j, M V}}{s-a_{j, j}}
$$

Para $A_{C R}$, têm-se as seguintes expressões para os ganhos estáticos:

$$
K_{x_{i}, M V}=\frac{M}{D} \frac{b_{i, M V}}{\text { Out }_{1, i}} ; \quad K_{x_{i}, x_{i-1}}=-\frac{I_{1, i-1}}{\text { Out }_{1, i}} ; \quad K_{x_{i-1}, x_{i}}=-\frac{I D_{1_{i-1, i}}}{\text { out }_{1 i-1, i-1}}
$$

\subsection{Estrutura do Modelo Dinâmico Simplificado Proposto}

O modelo proposto neste trabalho é linear, representado por uma matriz de transferência cujos elementos são funções de transferência FT de segunda ordem $\widetilde{G}_{Y, U}(s)$, em que $Y$ é a variável de saída e $U$ é a variável de entrada da respectiva FT.

$$
\left[\begin{array}{l}
\Delta x_{1}(s) \\
\Delta x_{n}(s)
\end{array}\right]=\left[\begin{array}{cc}
\tilde{G}_{x_{1}, L}(s) & \tilde{G}_{x_{1}, V}(s) \\
\tilde{G}_{x_{n}, L}(s) & \tilde{G}_{x_{n}, V}(s)
\end{array}\right]\left[\begin{array}{l}
\Delta L(s) \\
\Delta V(s)
\end{array}\right] ; \quad \tilde{G}_{Y, U}(s)=\frac{K_{Y, U}\left(\widetilde{\beta}_{Y, U} s+1\right)}{\left(\tilde{\tau}_{1} s+1\right)\left(\tilde{\tau}_{2} s+1\right)}
$$

\section{MODELO ESTÁTICO PROPOSTO}

Devido à estrutura tridiagonal de $A_{C}$, a solução analítica da matriz de ganhos estáticos $M_{K}$ pôde ser obtida a partir da aplicação do Método de Thomas ao seguinte sistema de equações linear formulado:

$$
A_{C} M_{K}=B_{C}
$$

Em função da forma iterativa da solução para $M_{K}$, é possível relacionar diretamente a expressão do ganho correspondente a uma determinada entrada sobre um estado como função de expressões de

ganho de subsistemas internos, devido ao formato apresentado pela solução, como expresso pelas Equações 44 a 46. 


$$
\begin{array}{ll}
K_{f b_{1}}=1 ; K_{f b_{i}}=\frac{1}{1-K_{f b_{i-1}} K_{x_{i}, x_{i-1}} K_{x_{i-1}, x_{i}}} & 1<i<n \\
K_{I n t_{x_{1}, M V}}=K_{x_{1}, M V} ; K_{I n t_{x_{i}, M V}}=K_{f b_{i}}\left(K_{x_{i}, M V}+K_{x_{i}, x_{i-1}} K_{I n t_{x_{i-1}, M V}}\right) & \\
K_{M x_{n}, M V}=K_{I n t_{x_{n}, M V}} & 1<i<n \\
K_{M x_{x_{i}, M V}}=K_{I n t_{x_{i}, M V}}+K_{f b_{i}} K_{x_{i}, x_{i+1}} K_{M x_{i+1}, M V} & \\
K_{M_{x_{1}, M V}}=K_{x_{1}, M V}+K_{x_{1}, x_{2}} K_{M_{x_{2}, M V}} &
\end{array}
$$

Os termos $K_{M_{x_{j}, M V}}$ correspondem aos ganhos estáticos globais da composição $x_{j}$ com respeito à respectiva $\mathrm{MV}$, e são os elementos de $M_{K}$.

\section{MODELO DINÂMICO SIMPLIFICADO PROPOSTO}

$\mathrm{O}$ autovetor $v_{1}$ correspondente ao autovalor dominante $\lambda_{1}$ de $A_{C}$ pode ser aproximado por $\tilde{v}_{1}$ através do Método das Potências (A). Este é eficiente para matrizes esparsas, pois não requer a execução de decomposições matriciais. A estrutura tridiagonal de $A_{C}$ satisfaz este critério. Contudo, o autovalor de interesse é o $n$, que é o de menor valor absoluto. Para tanto, os autovetores $\tilde{v}_{m}$ não dominantes, $2 \leq m \leq n$, podem ser aproximados por uma extensão de (A): o Método da Deflação com Complemento de Schur (B). A qualidade das aproximações dos componentes $\tilde{v}_{m_{i}}$ de $\tilde{v}_{m}$ por (A) e (B) depende de uma estimação inicial $\widetilde{w}_{m}$ para $\tilde{v}_{m}$. Essa pode ser obtida através de técnicas como o Método de Bandas de Autovalores Utilizando Traços (C) desenvolvido por Wolkowicz e Styan (1980). Este requer que $A_{C}$ seja positiva definida. O método proposto para estimação do autovalor dominante, baseado em uma combinação das técnicas (A), (B) e (C), é descrito a seguir.

Autovalores aproximados: A expressão para cada elemento $\tilde{v}_{m_{i}}$ de $\tilde{v}_{m}$ é obtida através de uma iteração do Método das Potências. Caso este seja aplicado com mais de uma iteração, as expressões para $\tilde{\lambda}_{m}$ são mais precisas, porém de complexidade crescente, tornando-se inviáveis para os propósitos deste trabalho. Para que apenas uma iteração forneça resultados satisfatórios, a aproximação $\widetilde{w}_{m}$ deve ser suficientemente precisa. Para tanto, o seguinte problema de autovalores aproximados foi formulado:

$$
A_{G n} \widetilde{w}_{m}=\tilde{\lambda}_{m_{0}} \widetilde{w}_{m}
$$

$A_{G n}$ é a matriz Jacobiana da representação em espaço de estados de $f b_{n}(s)$, definida por:

$$
f b_{n}(s)=\frac{1}{1-f b_{n-1}(s) G_{n, n-1}(s) G_{n-1, n}(s)}
$$

Isto se justifica porque os polos de $f b_{n}(s)$ são iguais aos autovalores de $A_{C}$. Para simplificar o tratamento matemático, $A_{G n}$ é representada na forma canônica de controlabilidade: 


$$
\left[\begin{array}{cccccc}
0 & 1 & 0 & \cdots & \cdots & 0 \\
0 & 0 & 1 & 0 & \cdots & \vdots \\
0 & 0 & 0 & 1 & \cdots & \vdots \\
\vdots & \vdots & \vdots & \vdots & \vdots & \vdots \\
\vdots & \vdots & \vdots & \vdots & \vdots & 1 \\
-\vartheta_{0} & -\vartheta_{1} & -\vartheta_{2} & -\vartheta_{3} & \cdots & -\vartheta_{n-1}
\end{array}\right]\left[\begin{array}{c}
\widetilde{w}_{m_{1}} \\
\widetilde{w}_{m_{2}} \\
\vdots \\
\vdots \\
\widetilde{w}_{m_{n}}
\end{array}\right]=\tilde{\lambda}_{0_{m}}\left[\begin{array}{c}
\widetilde{w}_{m_{1}} \\
\widetilde{w}_{m_{2}} \\
\vdots \\
\vdots \\
\widetilde{w}_{m_{n}}
\end{array}\right]
$$

Os elementos $\vartheta_{k}$ são os coeficientes do polinômio característico de $f b_{n}(s)$, cujas expressões são de exaustiva determinação. Portanto, aproxima-se a componente $\widetilde{w}_{m_{1}}$ por um valor unitário. Isto não prejudica a precisão do método, e o sistema de equações se torna:

$$
\widetilde{w}_{m_{i}}=\left(-\tilde{\lambda}_{0_{m}}\right)^{i-1} \quad 1<i \leq n
$$

Os autovalores $\tilde{\lambda}_{0_{m}}$ são estimações, definidos em função de valores limites aproximados para $\lambda_{m}$ a partir do Método de Bandas para Autovalores Utilizando Traços, de Wolkowicz e Styan (1980).

$$
\begin{array}{ll}
\tilde{\lambda}_{0_{m}}=\frac{1}{2}\left(\lambda_{m_{\text {sup }}}+\lambda_{m_{\text {inf }}}\right) ; & \lambda_{m_{\text {sup }}}=\bar{\lambda}+\sigma \sqrt{\frac{n-m}{n}} ; \lambda_{m_{\text {inf }}}=\bar{\lambda}-\sigma \sqrt{\frac{m-1}{n-(m-1)}} ; \\
\bar{\lambda}=\frac{1}{n} \operatorname{Tr}\left(A_{C}\right) ; & \sigma=\frac{1}{n}\left(n \operatorname{Tr}\left(A_{C}{ }^{2}\right)-\left(\operatorname{Tr}\left(A_{C}\right)\right)^{2}\right)^{1 / 2}
\end{array}
$$

Onde $\bar{\lambda}$ é a média dos autovalores de $A_{C}$ e $\sigma$ é o desvio padrão dos autovalores de $A_{C}$.

A partir do método apresentado, um modelo dinâmico simplificado analítico para colunas de destilação foi concebido. Este está representado em termos de variáveis de operação da coluna.

\section{Estimação dos polos:}

Expressões oriundas do Método das Potências com Deflação com Complemento de Schur.

$$
\begin{array}{ll}
d x 0_{m_{1}}=\frac{D}{M}\left(\text { out }_{m_{1,1}}+\operatorname{InDSum} 0_{m_{1}}\left(-\lambda_{0_{m}}\right)\right) & \\
d x 0_{m_{n}}=\frac{D}{M}\left(\text { out }_{m_{n, n}}\left(-\lambda_{0_{m}}\right)^{n-1}+\operatorname{InUSum} 0_{m_{n}}\right) & \\
d x 0_{m_{i}}=\frac{D}{M}\left(\text { out }_{m_{i, i}}\left(-\lambda_{0_{m}}\right)^{i-1}+\operatorname{InDSum} 0_{m_{i}}+\operatorname{InUSum} 0_{m_{i}}\right) & 1<i<n \\
d x 1_{m_{1}}=\frac{D}{M}\left(\text { out }_{m_{1,1}} d x 0_{m_{1}}+\operatorname{InDSum} 1_{m_{1}}\right) & \\
d x 1_{m_{n}}=\frac{D}{M}\left(\text { out }_{m_{n, n}} d x 0_{m_{n}}+\operatorname{InUSum} 1_{m_{n}}\right) & 1<i<n \\
d x 1_{m_{i}}=\frac{D}{M}\left(\text { out }_{m_{i, i}} d x 0_{m_{i}}+\operatorname{InDSum} 1_{m_{i}}+\operatorname{InUSum} 1_{m_{i}}\right) &
\end{array}
$$




$$
\begin{aligned}
& d x 0 \operatorname{Sum}_{m_{i}}=d x 0_{m_{j}} \text { Out }_{m_{j, j}}+\sum_{k=j}^{n}\left(d x 0_{m_{k}} \operatorname{InU_{m_{k,j}}}\right)+\sum_{k=1}^{j}\left(d x 0_{m_{k}} \operatorname{InD}_{m_{k, j}}\right) \\
& \operatorname{In} U_{m_{i, j}}=\operatorname{InU}_{m-1_{i, j}}+\frac{1}{\widetilde{\lambda}_{m-1} \sum_{k=1}^{n}\left(d x 0_{m-1}\right)^{2}}\left(d x 1_{m-1_{i}}\right)\left(d x 0 S u m_{m-1_{i}}\right) \quad j<i \\
& \operatorname{In} D_{m_{i, j}}=\operatorname{In} D_{m-1_{i, j}}+\frac{1}{\bar{\lambda}_{m-1} \sum_{k=1}^{n}\left(d x 0_{m-1}\right)^{2}}\left(d x 1_{m-1_{i}}\right)\left(d x 0 S u m_{m-1}\right) \quad j>i \\
& \text { Out }_{m_{i, j}}=\text { Out }_{m-1_{i, j}}+\frac{1}{\widetilde{\lambda}_{m-1} \sum_{k=1}^{n}\left(d x 0_{m-1}\right)^{2}}\left(d x 1_{m-1_{i}}\right)\left(d x 0 S u m_{m-1 i}\right) \quad j=i \\
& \operatorname{InDSum} 0_{m_{i}}=\sum_{k=i+1}^{n}\left(\operatorname{In} D_{m_{i, k}}\right)\left(-\lambda_{0_{m}}\right)^{k-1}, 1 \leq i<n \\
& \operatorname{InUSum} 0_{m_{i}}=\sum_{k=1}^{i-1}\left(\operatorname{In} U_{m_{i, k}}\right)\left(-\lambda_{0_{m}}\right)^{k-1}, \quad 1<i \leq n \\
& \lambda_{m_{i}}=-\frac{1}{\sum_{k=1}^{n}\left(d x 0_{m_{k}}\right)^{2}} \sum_{k=1}^{n}\left(d x 0_{m_{k}} d x 1_{m_{k}}\right)
\end{aligned}
$$

Determinação da Ordem Reduzida e Estimação do Zero: Através da análise modal de Litz, foi verificado que o sistema pode ser representado por dois modos dinâmicos dominantes, equivalentes a uma FT de ordem 2. Aplicando-se a redução de ordem de Litz, para a obtenção do zero, é necessário primeiramente estimar a inversa $d x 4$ da matriz composta pelos vetores $d x 0_{m}$. A obtenção de $d x 4$ é realizada conforme o seguinte procedimento baseado no método de Leverrier-Fadeev:

$$
\begin{aligned}
& d x 0_{m}=\frac{d x 0_{m}}{\sqrt{\sum_{i=1}^{n}\left(d x 0_{m_{i}}\right)^{2}}} ; d x 3_{1_{i, j}}=d x 0_{n-j+1_{i}} \\
& d x 3 \text { Sum }_{m_{i, j}}=\sum_{i=1}^{n} d x 3_{m_{i, i}}, i=j ; \quad d x 3 \text { Sum }_{m_{i, j}}=0, i \neq j \\
& d x 3_{m_{i, j}}=\sum_{k=1}^{n} d x 3_{1_{i, k}}\left(d x 3_{m-1_{k, j}}-d x 3 \text { Sum }_{m-1_{k, j}}\right), \quad 2 \leq m \leq n \\
& d x 4_{i, j}=\frac{1}{d x 3 \text { Sum }_{n_{n, n}}}\left(d x 3_{n-1_{i, j}}-d x 3 \text { Sum }_{m-1_{i, j}}\right)
\end{aligned}
$$

Como há $D O M=2$ modos dominantes, da redução modal de Litz, para $1 \leq i \leq D O M$ :

$$
\begin{aligned}
& d x 3 D O M_{i}=d x 3_{1_{1, i}} ; \quad d x 4 D O M_{i}=\sum_{j=1}^{n} b_{j, M V} d x 4_{i, j} \\
& z=\frac{d x 3 D O M_{1} d x 4 D O M_{1}+d x 3 D O M_{2} d x 4 D O M_{2}}{d x 3 D O M_{1} d x 4 D O M_{1}+d x 3 D O M_{2} d x 4 D O M_{2}} ; \quad \beta=-\frac{1}{z}
\end{aligned}
$$

Função de Transferência Final: 


$$
G=\frac{K(\beta s+1)}{\left(-\frac{1}{\lambda_{n}} s+1\right)\left(-\frac{1}{\lambda_{n-1}} s+1\right)}
$$

\section{RESULTADOS, DISCUSSÕES E CONCLUSÕES}

Uma coluna binária de alta pureza, com $\mathrm{N}_{\mathrm{T}}$ estágios alimentada em $\mathrm{F}_{\mathrm{T}}$, adaptada da coluna "A" do trabalho de Skogestad (1987), é o caso de estudo. Os dados do sistema são (vazões em $\mathrm{kmol} \mathrm{min}^{-1}$ ):

Tabela 1 - Dados da Coluna Estudada

\begin{tabular}{|l|l|c|c|c|c|c|c|c|c|c|c|c|}
\hline $\mathrm{N}_{\mathrm{T}}$ & $\mathrm{F}_{\mathrm{T}}$ & $\mathrm{F}$ & $\mathrm{V}$ & $\mathrm{L}$ & $\mathrm{q}$ & $\mathrm{R}_{\mathrm{LD}}$ & $\mathrm{R}_{\mathrm{DF}}$ & $\alpha$ & $\mathrm{M}$ & $\mathrm{M}_{\mathrm{C}}$ & $\mathrm{M}_{\mathrm{R}}$ & $\mathrm{X}_{\mathrm{f}}$ \\
\hline 41 & 21 & 1 & 3,206 & 2,706 & 1 & 5,41 & 0,499 & 2,5 & 0,5 & 32,1 & 10 & 0,5 \\
\hline
\end{tabular}

O polo de menor valor absoluto, correspondente à constante de tempo dominante, foi estimado:

Tabela 2 - Polo Dominante $\left(s^{-1}\right)$ Estimado

\begin{tabular}{|c|c|}
\hline Característica & $\lambda_{n}$ \\
\hline Valor Real & -0.0000182236 \\
\hline Valor Estimado & -0.0000184027 \\
\hline Erro Relativo (\%) & 0.98 \\
\hline
\end{tabular}

A predição da dinâmica dominante de sistemas de elevada crucial revelou-se satisfatória para fins de modelos lineares simplificados. Modelos de baixa ordem podem ser elaborados a partir da metodologia proposta, que apresenta uma solução analítica em termos de variáveis operacionais da coluna de simples e rápida implementação. As soluções de ganhos estáticos foram exatas com relação ao modelo linear original de alta ordem. Isto se explica porque os valores de ganho são obtidas analiticamente através do método proposto. Diversos sistemas da literatura requerem métodos empíricos para a determinação do modelo estático, os quais se restringem a específicas faixas operacionais. Contudo, o método proposto para o modelo estático proporciona soluções exatas para sistemas cuja matriz Jacobiana de estados seja tridiagonal ou de maior esparsidade.

\section{REFERÊNCIAS}

LEVY, R.S., Foss, A.S., GRENS, E.A, Response modes of a binary distillation column. Ind. Eng. Chem. Fundamentals, 8, 765-776, DOI:10.1021/i160032a027,1969.

SKOGESTAD, S., MORARI, M., The dominant time constant for distillation columns. Comput. Chem. Engng., 11, 607-617, DOI: 98-1354(87)87006-0. 1987.

BENALlOU, A., SEBORG, D.E. MELLICHAMP, D.A., Dynamic compartmental models for separation processes. AlChE Journal,32,1067-1078, 1986.

WOLKOWICZ, H., STYAN, G.P.H. Bounds for Eigenvalues Using Traces. Linear Algebra and its Applications. 29,471-506, 1980. 